\title{
Neurohumoral effects of aliskiren in patients with symptomatic heart failure receiving a mineralocorticoid receptor antagonist: the Aliskiren Observation of Heart Failure Treatment study
}

\author{
Bertram Pitt ${ }^{1}$, Roberto Latini ${ }^{2}$, Aldo P. Maggioni ${ }^{3}$, Scott D. Solomon ${ }^{4}$, \\ Beverly A. Smith ${ }^{5}$, Melanie Wright ${ }^{5}$, Margaret F. Prescott ${ }^{6}$, \\ and John J.V. McMurray 4,7*
}

${ }^{1}$ University of Michigan, Ann Arbor, MI, USA; ${ }^{2}$ Department of Cardiovascular Research, Istituto di Ricerche Farmacologiche Mario Negri, Milan, Italy; ${ }^{3}$ ANMCO Research Center, Florence, Italy; ${ }^{4}$ Brigham \& Women's Hospital, Boston, MA, USA; ${ }^{5}$ Novartis Pharma AG, Basel, Switzerland; ${ }^{6}$ Novartis Pharmaceutical Corporation, East Hanover, NJ, USA; and ${ }^{7}$ British Heart Foundation Cardiovascular and Research Centre, University of Glasgow, 126 University Place, Glasgow G12 8TA, UK

Received 19 November 2010; revised 1 February 2011; accepted 4 February 2011; online publish-ahead-of-print 4 April 2011

\begin{abstract}
Aims
We evaluated the influence of concomitant mineralocorticoid receptor antagonists (MRAs) on the safety and neurohumoral effects of a direct renin inhibitor in the ALiskiren Observation of Heart Failure Treatment (ALOFT) study.

Methods Patients with stable New York Heart Association class II-IV heart failure (HF), plasma B-type natriuretic peptide and results (BNP) concentration $>100 \mathrm{pg} / \mathrm{mL}$, and treated with an angiotensin-converting enzyme inhibitor (or angiotensin receptor blocker) and $\beta$-blocker were randomized to once-daily, double-blind treatment with aliskiren $150 \mathrm{mg}$ or placebo, added to optimal HF therapy, for 12 weeks. Safety, tolerability, and effects of aliskiren on neurohumoral biomarkers were assessed in patients who received (MRA+) and did not receive (MRA-) MRA treatment at baseline. Of the 302 randomized patients, 101 were receiving MRA treatment (aliskiren, $n=52$; placebo, $n=49$ ). Mineralocorticoid receptor antagonist status did not affect the ability of aliskiren $150 \mathrm{mg}$, added to standard HF therapy, to lower BNP, N-terminal proBNP, plasma renin activity, and urinary aldosterone. For example, the end-of-study to baseline ratio of geometric mean for BNP was: MRA+ group: aliskiren 0.68 [95\% confidence interval $(\mathrm{Cl}) 0.47$, 0.98], placebo 0.85 (0.58, 1.24); MRA - group: aliskiren $0.62(0.45,0.84)$, placebo $0.85(0.63,1.15)$, interaction $P=0.720$. The incidence of pre-specified adverse events (renal dysfunction, symptomatic hypotension, and hyperkalaemia) was low, and there were no significant differences between aliskiren and placebo in either MRA subgroup.

Conclusion Aliskiren $150 \mathrm{mg}$ added to standard HF therapy was well tolerated over 12 weeks and provided beneficial changes in neurohumoral biomarkers regardless of concomitant MRA treatment.

Keywords

Aliskiren • B-type natriuretic peptide • Mineralocorticoid receptor antagonist • Renin
\end{abstract}

\section{Introduction}

The ALiskiren Observation of Heart Failure Treatment (ALOFT) study was a randomized, placebo-controlled study in 302 patients with symptomatic heart failure (HF). The study assessed the safety and neurohumoral effects of adding the direct renin inhibitor (DRI) aliskiren $150 \mathrm{mg}$ once daily to standard therapy including an angiotensin-converting enzyme (ACE) inhibitor [or angiotensin receptor blocker (ARB), but not both] and a $\beta$-blocker. ${ }^{1}$ ALOFT showed that aliskiren was effective in reducing levels of plasma

* Corresponding author. Tel: +44 141330 3479, Fax: +44 141330 6955, Email: john.mcmurray@glasgow.ac.uk

Published on behalf of the European Society of Cardiology. All rights reserved. (C) The Author 2011. For permissions please email: journals.permissions@oup.com. 
B-type natriuretic peptide (BNP) and plasma N-terminal prohormone BNP (NT-proBNP), and was generally well tolerated over a 12-week period. Approximately one-third of patients in ALOFT were receiving a mineralocorticoid receptor antagonist (MRA) at baseline before being randomized to receive aliskiren or placebo, and could therefore have received combination treatment with three agents acting on the renin-angiotensin-aldosterone system (RAAS), i.e. an ACE inhibitor or ARB, MRA, and DRI (or even four agents if the renin-suppressing action of $\beta$-blockers is considered). The use of MRAs is likely to increase as a result of the findings of the recently reported Eplerenone in Mild Patients Hospitalization And Survlval Study in Heart Failure (EMPHASIS-HF) study. ${ }^{2,3}$ In view of this and the concern about the safety of using multiple RAAS inhibitors, ${ }^{4,5}$ a post-hoc subgroup analysis of ALOFT was undertaken to provide further insight into the potential efficacy and safety of adding aliskiren to the regimen of patients already receiving multiple RAAS inhibitors. This analysis is particularly relevant in the light of a major ongoing mortality and morbidity trial, Aliskiren Trial to Minimize OutcomeS in Patients with HEart failuRE (ATMOSPHERE) in which aliskiren is being added to conventional therapy, including, if the investigator chooses, an MRA. ${ }^{6}$

\section{Methods}

This was a post-hoc subgroup analysis of data from the ALOFT trial, a multicentre, randomized, double-blind, placebo-controlled study in patients with symptomatic HF that has been described in detail elsewhere. $^{1}$ This subgroup analysis evaluated patients according to whether they had or had not received an MRA as part of their standard HF therapy at baseline (MRA + and MRA - subgroups, respectively). The study was performed in accordance with the International Conference on Harmonisation Guideline for Good Clinical Practice and the Declaration of Helsinki, and approved by the local and central ethical review boards, and all patients provided written informed consent.

\section{Patients}

Men and women aged $\geq 18$ years with stable HF (New York Heart Association classes II-IV), a current or past diagnosis of hypertension, plasma BNP concentration $>100 \mathrm{pg} / \mathrm{mL}(>28.9 \mathrm{pmol} / \mathrm{L})$, and receiving a stable dose of an ACE inhibitor (or ARB) and a $\beta$-blocker (unless contraindicated or not tolerated) were eligible for inclusion. Patients receiving an ACE inhibitor in combination with an ARB were excluded from the study. Other key exclusion criteria included: HF related to obstructive valve disease or hypertrophic, restrictive, or infective cardiomyopathy; pregnancy; lung disease; systolic blood pressure $<90 \mathrm{mmHg}$; and myocardial infarction, cerebrovascular accident, transient ischaemic attack, or coronary revascularization within 6 months of initiating screening.

\section{Study design and assessments}

Following a 2-week, single-blind, placebo run-in period to establish eligibility, patients were randomized equally to receive once-daily treatment with either aliskiren $150 \mathrm{mg}$ or placebo for 12 weeks, in addition to their current HF therapy. Randomization was stratified according to left ventricular ejection fraction (LVEF) at baseline (LVEF $>40 \%$ and $\leq 40 \%$ ), and patients were evaluated at baseline and 12 weeks after randomization.
The primary objective of this study was to evaluate the safety and tolerability of aliskiren, specifically the incidence of the pre-defined adverse events of renal dysfunction [elevation of serum creatinine levels to $>3.0 \mathrm{mg} / \mathrm{dL}(>265 \mu \mathrm{mol} / \mathrm{L})$ or other adverse events related to renal dysfunction], hyperkalaemia (serum potassium levels $>5.5 \mathrm{mmol} / \mathrm{L}$ or other adverse events related to hyperkalaemia) and symptomatic hypotension.

Efficacy endpoints included changes from baseline to the end of the study in levels of plasma NT-proBNP and other neurohumoral biomarkers, such as plasma BNP, plasma renin activity (PRA), plasma renin concentration (PRC) and urinary aldosterone, and mean sitting systolic and diastolic blood pressure ( $\mathrm{msSBP}$ and msDBP). Echocardiograms were performed at the screening and Week 12 assessments, and were used to evaluate changes in cardiac size and left ventricular function.

Plasma renin activity was measured by means of radioimmunoassay of generated angiotensin I (DiaSorin kit; DiaSorin, Stillwater, MN, USA), of which the lower limit of quantification (LLOQ) was $0.2 \mathrm{ng} /$ $\mathrm{mL} / \mathrm{h}$. Plasma renin concentration was measured by immunoradiometry using a solid-phase sandwich assay (Renin III Generation, CIS Bio International, Gif-sur-Yvette, France; LLOQ 1.0 pg/mL), as described initially by Ménard and co-workers and subsequently modified by Nussberger and colleagues. Plasma BNP concentration was measured using a two-site dual-monoclonal immunochemiluminescent assay (ADVIA Centaur BNP assay, Siemens Centaur XP, Malvern, PA, USA, Diagnostics Division). Plasma NT-proBNP concentration was measured using a chemiluminescent immunometric assay (Roche Diagnostics NT-proBNP Assay on ELECSYS 2010, Indianapolis, IN, USA). Total urinary aldosterone level was measured by radioimmunoassay (Siemens, Deerfield, IL, USA) following acidification with $3.2 \mathrm{~N} \mathrm{HCl}$ and incubation for $24 \mathrm{~h}$ at room temperature in the dark.

All biomarkers were measured at Clinical Research Laboratories, Medinet in Breda, the Netherlands, or Lenexa, KS, USA. The coefficients of variation for the assays were as follows: PRA-17.1\% at $0.20 \mathrm{ng} / \mathrm{mL} / \mathrm{h}$ to $5.3 \%$ at $8.83 \mathrm{ng} / \mathrm{mL} / \mathrm{h}$; PRC $-9.24 \%$ at $4.49 \mathrm{pg} / \mathrm{mL}$ to $7.22 \%$ at $43.29 \mathrm{pg} / \mathrm{mL}$; BNP-5.85\% at $39.2 \mathrm{pg} / \mathrm{mL}$ to $3.95 \%$ at $1535 \mathrm{pg} / \mathrm{mL}$; NT-proBNP-3.6\% at $131 \mathrm{pg} / \mathrm{mL}$ to $3.1 \%$ at $4259 \mathrm{pg} /$ $\mathrm{mL}$; urine aldosterone $-7.2 \%$ at $15.8 \mu \mathrm{g} / \mathrm{L}$ to $5.5 \%$ at $78.5 \mu \mathrm{g} / \mathrm{L}$.

\section{Statistical analysis}

Baseline parameters were compared for differences between treatment groups within the MRA+ and MRA - subgroups, using the $t$-test statistic for continuous characteristics and the $\chi^{2}$ statistic (Fisher's exact test when any particular expected count was $\leq 5$ ) for categorical characteristics. Between-treatment comparisons using least-squares means were performed for aliskiren vs. placebo in the overall population and within each of the MRA+ and MRA - subgroups. In order to perform the subgroup analysis, changes from baseline to endpoint (Week 12) were analysed using an analysis of covariance (ANCOVA) model with treatment, region, LVEF ( $>40$ or $\leq 40 \%$ ) and subgroup as factors, baseline as a covariate and treatment by subgroup as an interaction. A logtransformation was applied before analysis of the neurohumoral parameters (NT-proBNP, BNP, PRA, PRC, and urinary aldosterone). Treatment comparisons for the primary safety parameters, and the incidence of adverse events and laboratory abnormalities within each subgroup were analysed using Fisher's exact test or the $\chi^{2}$ test. All statistical analyses were performed using SAS version 8.2 (or higher). 
Table I Baseline demographic, clinical, and disease characteristics (intent-to-treat population)

\begin{tabular}{|c|c|c|c|c|c|c|}
\hline & \multicolumn{2}{|c|}{ Overall population } & \multicolumn{2}{|c|}{ Not MRA treated (MRA-) } & \multicolumn{2}{|c|}{ MRA treated $($ MRA +$)$} \\
\hline & $\begin{array}{l}\text { Placebo } \\
(n=146)\end{array}$ & $\begin{array}{l}\text { Aliskiren } 150 \mathrm{mg} \\
(n=156)\end{array}$ & $\begin{array}{l}\text { Placebo } \\
(n=97)\end{array}$ & $\begin{array}{l}\text { Aliskiren } 150 \mathrm{mg} \\
(n=104)\end{array}$ & $\begin{array}{l}\text { Placebo } \\
(n=49)\end{array}$ & $\begin{array}{l}\text { Aliskiren } 150 \mathrm{mg} \\
(n=52)\end{array}$ \\
\hline \multicolumn{7}{|l|}{ Demographic characteristics } \\
\hline Age, year & $68.4 \pm 10.2$ & $67.4 \pm 10.6$ & $70.2 \pm 9.8$ & $68.4 \pm 10.4$ & $64.9 \pm 10.2$ & $65.3 \pm 10.8$ \\
\hline Male, $n(\%)$ & $111(76.0)$ & $125(80.1)$ & $81(83.5)$ & $84(80.8)$ & $30(61.2)$ & $41(78.8)$ \\
\hline Caucasian, $n$ (\%) & $144(98.6)$ & $150(96.2)$ & $97(100)$ & $101(97.1)$ & $47(95.9)$ & $49(94.2)$ \\
\hline \multicolumn{7}{|l|}{ Clinical characteristics } \\
\hline eGFR, $\mathrm{mL} / \mathrm{min} / 1.73 \mathrm{~m}^{2}$ & $67.8 \pm 19.1$ & $70.0 \pm 21.3$ & $68.4 \pm 20.7$ & $69.2 \pm 20.5$ & $66.4 \pm 15.8$ & $71.7 \pm 22.9$ \\
\hline $\mathrm{BMI} \geq 30 \mathrm{~kg} / \mathrm{m}^{2}, \mathrm{n}(\%)$ & $33(22.6)$ & $42(26.9)$ & $19(19.6)$ & $25(24.0)$ & $14(28.6)$ & $17(32.7)$ \\
\hline \multicolumn{7}{|l|}{ Disease characteristics } \\
\hline \multicolumn{7}{|l|}{ Heart failure } \\
\hline $\begin{array}{l}\text { Time since } \\
\text { diagnosis, years }\end{array}$ & $4.9 \pm 5.3$ & $4.1 \pm 3.9$ & $4.7 \pm 5.7$ & $3.9 \pm 3.7$ & $5.1 \pm 4.6$ & $4.3 \pm 4.3$ \\
\hline \multicolumn{7}{|l|}{ Aetiology, n (\%) } \\
\hline Ischaemic & $79(54.1)$ & $86(55.1)$ & $56(57.7)$ & $61(58.7)$ & $23(46.9)$ & $25(48.1)$ \\
\hline $\begin{array}{l}\text { Idiopathic } \\
\text { cardiomyopathy }\end{array}$ & $29(19.9)$ & $36(23.1)$ & $18(18.6)$ & $25(24.0)$ & $11(22.4)$ & $11(21.2)$ \\
\hline Hypertensive & $25(17.1)$ & $25(16.0)$ & $18(18.6)$ & $15(14.4)$ & $7(14.3)$ & $10(19.2)$ \\
\hline Other & $13(8.9)$ & $9(5.8)$ & $5(5.2)$ & $3(2.9)$ & $8(16.3)$ & $6(11.5)$ \\
\hline LVEF $\leq 40 \%, n(\%)^{\mathrm{a}}$ & $122(92.4)$ & $132(95.0)$ & $77(79.4)$ & $90(86.5)$ & $45(91.8)$ & $42(80.8)$ \\
\hline \multicolumn{7}{|l|}{ NYHA class, n (\%) } \\
\hline I & $1(0.7)$ & 0 & $1(1.0)$ & 0 & 0 & 0 \\
\hline ॥ & $87(59.6)$ & $98(62.8)$ & $58(59.8)$ & $69(66.3)$ & $29(59.2)$ & $29(55.8)$ \\
\hline III & $58(39.7)$ & $56(35.9)$ & $38(39.2)$ & $34(32.7)$ & $20(40.8)$ & $22(42.3)$ \\
\hline IV & 0 & $2(1.3)$ & 0 & $1(1.0)$ & 0 & $1(1.9)$ \\
\hline $\begin{array}{l}\text { Duration of } \\
\text { hypertension, years }\end{array}$ & $12.3 \pm 9.6$ & $11.4 \pm 8.7$ & $12.8 \pm 10.2$ & $10.5 \pm 8.6$ & $11.2 \pm 8.4$ & $13.3 \pm 8.7$ \\
\hline Diabetes, $n(\%)$ & $49(33.6)$ & $57(36.5)$ & $36(37.1)$ & $38(36.5)$ & $13(26.5)$ & $19(36.5)$ \\
\hline \multicolumn{7}{|l|}{ Concomitant medication } \\
\hline ACE inhibitor, $n(\%)$ & $123(84.2)$ & $130(83.3)$ & 77 (79.4) & 85 (81.7) & $46(93.9)$ & $45(86.5)$ \\
\hline ARB, $n(\%)$ & $21(14.4)$ & $25(16.0)$ & $18(18.6)$ & $19(18.3)$ & $3(6.1)$ & $6(11.5)$ \\
\hline$\beta$-blocker, $n$ (\%) & $138(94.5)$ & $147(94.2)$ & $92(94.8)$ & $96(92.3)$ & $46(93.9)$ & $51(98.1)$ \\
\hline
\end{tabular}

All values are mean $\pm \mathrm{SD}$ unless otherwise stated.

ACE, angiotensin-converting enzyme; ARB, angiotensin receptor blocker; BMI, body mass index; eGFR, estimated glomerular filtration rate; LVEF, left ventricular ejection fraction; MRA, mineralocorticoid receptor antagonist; NYHA, New York Heart Association; SD, standard deviation.

${ }^{a}$ As measured by core echocardiography laboratory.

\section{Results}

Following the 2-week placebo run-in period, 302 patients were randomized to once-daily treatment for 12 weeks with aliskiren $150 \mathrm{mg}(n=156)$ or placebo $(n=146)$, in addition to standard therapy for HF (ACE inhibitor or ARB, plus $\beta$-blocker). In total, 277 patients $(91.7 \%)$ completed the double-blind treatment phase. A total of 101 patients (aliskiren, $n=52$; placebo, $n=49$ ) were receiving MRA treatment at baseline as part of their background HF therapy.

\section{Baseline patient characteristics}

Patient characteristics were generally well matched between the aliskiren and placebo treatment arms within each of the MRA - and
MRA+ subgroups (Table 1). Patients in the MRA+ subgroup tended to be younger than patients in the MRA - subgroup (mean age of 65 vs. 69 years, respectively) and a greater proportion of them (31 vs. $22 \%$, respectively) were obese [body mass index $\left.(\mathrm{BMI}) \geq 30 \mathrm{~kg} / \mathrm{m}^{2}\right]$. There was a lower proportion of men (70 vs. $82 \%)$ and a lower proportion of patients with ischaemic heart disease (48vs. $58 \%$ ) in the MRA + subgroup than in the MRA - subgroup, respectively. The proportion of patients receiving an ACE inhibitor was slightly higher in the MRA+ subgroup than in the MRA - subgroup (90 vs. $81 \%$, respectively). The most commonly used ACE inhibitors were ramipril $(n=88)$, enalapril $(n=60)$, lisinopril $(n=33)$, and perindopril $(n=28)$. The median daily doses in the aliskiren and placebo groups, respectively, were ramipril (5 vs. $5 \mathrm{mg}$ ), enalapril (20 vs. $10 \mathrm{mg}$ ), lisinopril (20 vs. 
Table 2 Baseline neurohumoral and echocardiographic parameters (intent-to-treat population)

\begin{tabular}{|c|c|c|c|c|c|c|}
\hline & \multicolumn{2}{|c|}{ Overall population } & \multicolumn{2}{|c|}{ Not MRA treated (MRA-) } & \multicolumn{2}{|c|}{ MRA treated $(\mathrm{MRA}+)$} \\
\hline & $\begin{array}{l}\text { Placebo } \\
(N=146)\end{array}$ & $\begin{array}{l}\text { Aliskiren } 150 \mathrm{mg} \\
(\mathrm{N}=156)\end{array}$ & $\begin{array}{l}\text { Placebo } \\
(N=97)\end{array}$ & $\begin{array}{l}\text { Aliskiren } 150 \mathrm{mg} \\
(\mathrm{N}=104)\end{array}$ & $\begin{array}{l}\text { Placebo } \\
(N=49)\end{array}$ & $\begin{array}{l}\text { Aliskiren } 150 \mathrm{mg} \\
(\mathrm{N}=52)\end{array}$ \\
\hline \multicolumn{7}{|c|}{ Neurohumoral parameters } \\
\hline \multirow[t]{3}{*}{ Plasma BNP, pg/mL } & {$[n=145]$} & {$[n=154]$} & {$[n=96]$} & {$[n=102]$} & {$[n=49]$} & {$[n=52]$} \\
\hline & 191.5 & 204.8 & 182.4 & 194.9 & 210.9 & 225.6 \\
\hline & $(165.2,222.1)$ & $(175.0,239.6)$ & $(149.8,222.0)$ & $(160.1,237.2)$ & $(168.8,263.5)$ & $(171.5,296.8)$ \\
\hline \multirow{3}{*}{$\begin{array}{l}\text { Plasma } \\
\text { NT-proBNP, } \\
\text { pg/mL }\end{array}$} & {$[n=141]$} & {$[n=147]$} & {$[n=93]$} & {$[n=95]$} & {$[n=48]$} & {$[n=52]$} \\
\hline & 1217 & 1387 & 1125 & 1244 & 1417 & 1690 \\
\hline & $(1002,1479)$ & $(1168,1647)$ & $(870,1457)$ & $(998,1551)$ & $(1055,1903)$ & $(1275,2240)$ \\
\hline \multirow[t]{2}{*}{ PRA, ng/mL/h } & {$[n=138]$} & {$[n=146]$} & {$[n=94]$} & {$[n=96]$} & {$[n=44]$} & {$[n=50]$} \\
\hline & $2.2(1.7,3.0)$ & $1.8(1.3,2.4)$ & $1.8(1.2,2.6)$ & $1.4(1.0,2.1)$ & $3.8(2.3,6.2)$ & $2.8(1.6,4.8)$ \\
\hline \multirow[t]{3}{*}{ PRC, ng/L } & {$[n=134]$} & {$[n=144]$} & {$[n=93]$} & {$[n=94]$} & {$[n=41]$} & {$[n=50]$} \\
\hline & 30.8 & 25.2 & 25.6 & 21.0 & 46.5 & 35.7 \\
\hline & $(24.2,39.1)$ & $(19.9,32.0)$ & $(19.0,34.5)$ & $(16.0,27.5)$ & $(30.9,70.2)$ & $(22.5,56.8)$ \\
\hline \multirow{3}{*}{$\begin{array}{l}\text { Urinary } \\
\text { aldosterone, } \\
\text { nmol/day }\end{array}$} & {$[n=131]$} & {$[n=145]$} & {$[n=89]$} & {$[n=97]$} & {$[n=42]$} & {$[n=48]$} \\
\hline & 22.7 & 24.3 & 22.3 & 23.0 & 23.7 & 27.1 \\
\hline & $(19.0,27.1)$ & $(20.8,28.4)$ & $(18.0,27.6)$ & $(18.9,28.0)$ & $(16.9,33.3)$ & $(20.7,35.6)$ \\
\hline \multicolumn{7}{|c|}{ Echocardiographic parameters } \\
\hline \multirow[t]{2}{*}{ MR/LA, \% } & {$[n=97]$} & {$[n=101]$} & {$[n=63]$} & {$[n=66]$} & {$[n=34]$} & {$[n=35]$} \\
\hline & $29.5 \pm 13.1$ & $30.2 \pm 13.8$ & $29.3 \pm 13.2$ & $29.7 \pm 12.3$ & $29.9 \pm 13.1$ & $31.1 \pm 16.5$ \\
\hline \multirow[t]{2}{*}{ LVEF, \% ${ }^{\mathrm{a}}$} & {$[n=132]$} & {$[n=139]$} & {$[n=85]$} & {$[n=95]$} & {$[n=47]$} & {$[n=44]$} \\
\hline & $31.1 \pm 5.5$ & $30.6 \pm 5.5$ & $31.9 \pm 5.3$ & $31.0 \pm 5.3$ & $29.6 \pm 5.4$ & $29.6 \pm 5.8$ \\
\hline Blood pressure & {$[n=146]$} & {$[n=156]$} & {$[n=97]$} & {$[n=104]$} & {$[n=49]$} & {$[n=52]$} \\
\hline $\mathrm{msSBP}, \mathrm{mmHg}$ & $127.6 \pm 16.4$ & $130.2 \pm 18.3$ & $128.8 \pm 16.5$ & $133.0 \pm 17.2$ & $125.1 \pm 16.1$ & $124.7 \pm 19.2$ \\
\hline $\mathrm{msDBP}, \mathrm{mmHg}$ & $76.4 \pm 8.4$ & $78.1 \pm 10.4$ & $77.1 \pm 8.4$ & $78.5 \pm 10.0$ & $75.1 \pm 8.5$ & $77.1 \pm 11.3$ \\
\hline
\end{tabular}

$12.5 \mathrm{mg}$ ), and perindopril (4 vs. $2 \mathrm{mg}$ ). In the aliskiren group at baseline, 8 patients were treated with potassium canrenoate $25 \mathrm{mg}$ daily; 2 with eplerenone $25 \mathrm{mg}$, and 1 with $50 \mathrm{mg}$ daily; 5 with spironolactone $12.5 \mathrm{mg}, 23$ with $25 \mathrm{mg}, 1$ with $37.5 \mathrm{mg}, 11$ with $50 \mathrm{mg}$, and 2 with $100 \mathrm{mg}$ daily. In the placebo group, 4 patients were treated with potassium canrenoate $25 \mathrm{mg}, 6$ with $50 \mathrm{mg}$, and 1 with $125 \mathrm{mg}$ daily; none with eplerenone and 2 with spironolactone $12.5 \mathrm{mg}, 26$ with $25 \mathrm{mg}, 9$ with $50 \mathrm{mg}$, and 1 with $100 \mathrm{mg}$ daily.

Baseline neurohumoral and echocardiographic parameters were also well matched between aliskiren and placebo treatment arms within each MRA subgroup (Table 2). Patients in the MRA+ subgroup had higher geometric mean baseline PRA (aliskiren 2.8, placebo $3.8 \mathrm{ng} / \mathrm{mL} / \mathrm{h}$ ) and PRC (aliskiren 35.7, placebo $46.5 \mathrm{ng} / \mathrm{L}$ ) than those in the MRA - subgroup (PRA: aliskiren 1.4, placebo $1.8 \mathrm{ng} / \mathrm{mL} / \mathrm{h}$; PRC: aliskiren 21.0, placebo $25.6 \mathrm{ng} / \mathrm{L})$.

\section{Changes in neurohumoral parameters}

In the overall population, aliskiren $150 \mathrm{mg}$ added to standard HF therapy significantly reduced geometric mean BNP (36\%), NT-proBNP (27\%), PRA (82\%), and urinary aldosterone (19\%) levels compared with placebo, and significantly increased PRC (161\%) (Figure 1A-E). Changes in neurohumoral parameters with aliskiren compared with placebo were similar in the MRA+ and MRA - subgroups; ANCOVA revealed no significant interaction between MRA treatment and the effect of aliskiren for any neurohumoral parameter (Figure 3A). Although there were modest differences in geometric mean reductions with aliskiren relative to placebo in plasma NT-proBNP in the MRA+ subgroup relative to the MRA - subgroup, these may be attributed to differences in baseline levels; 95\% confidence intervals for the two subgroups were completely overlapping and there was no significant interaction with MRA treatment status (Figure 3A). 

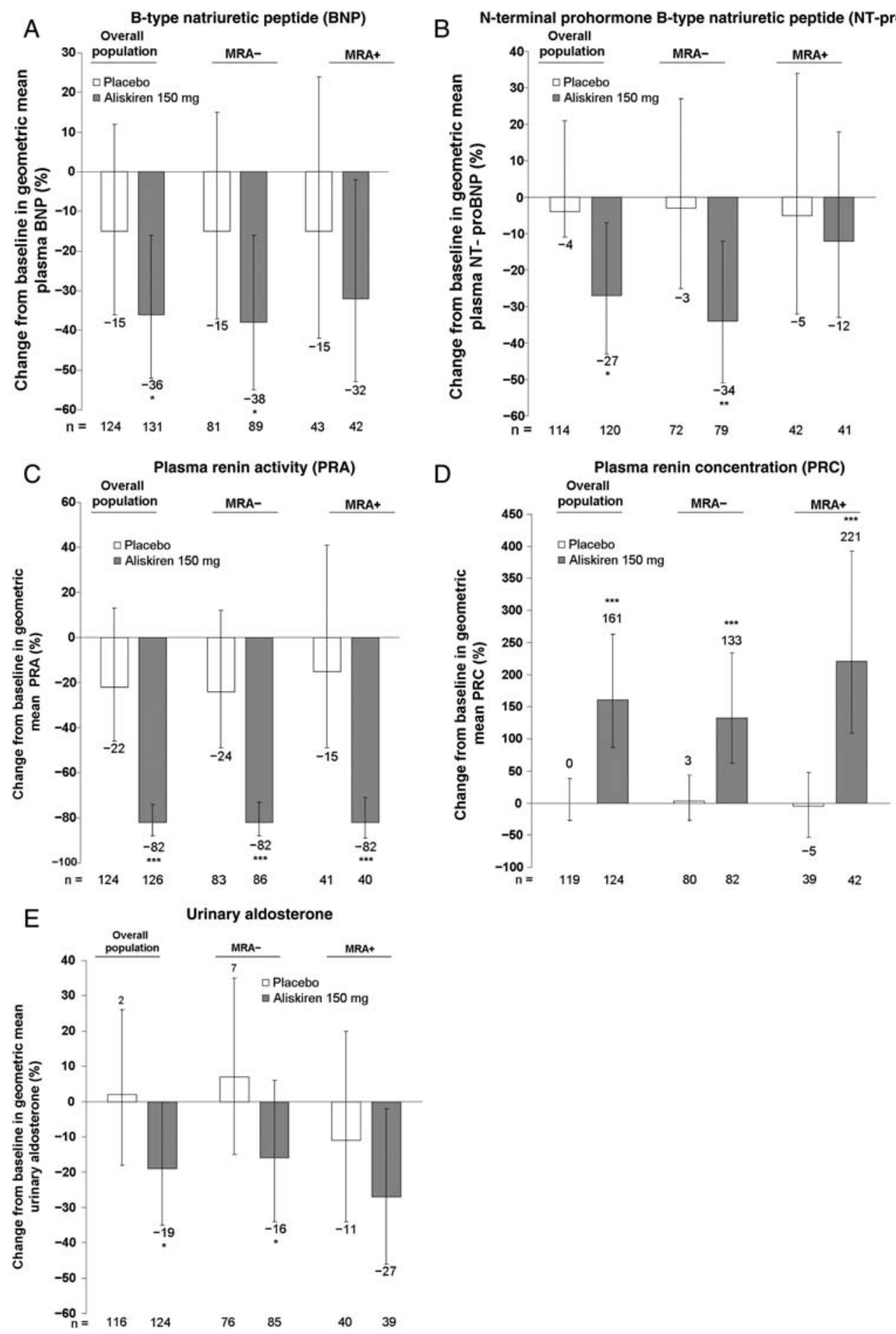

Figure I Changes from baseline in neurohumoral biomarkers according to mineralocorticoid receptor antagonist treatment status. (A) B-type natriuretic peptide, $(B) \mathrm{N}$-terminal prohormone B-type natriuretic peptide, $(C)$ plasma renin activity, $(D)$ plasma renin concentration, and $(E)$ urinary aldosterone. Data represent $(A-E)$ percentage change from baseline in geometric mean $(95 \% \mathrm{Cl})$ in the overall population and the subgroups of patients receiving (MRA+) or not receiving (MRA-) MRA treatment during the study. $P$ values were calculated using a two-way ANCOVA model with treatment, region, LVEF (>40 or $\leq 40 \%)$ and subgroup as factors, baseline as a covariate and treatment by subgroup as an interaction. $* P<0.05$ vs. placebo; $* * P<0.005$ vs. placebo; $* * * P<0.0001$ vs. placebo. ANCOVA, analysis of covariance; $\mathrm{Cl}$, confidence interval; LVEF, left ventricular ejection fraction; MRA, mineralocorticoid receptor antagonist. 

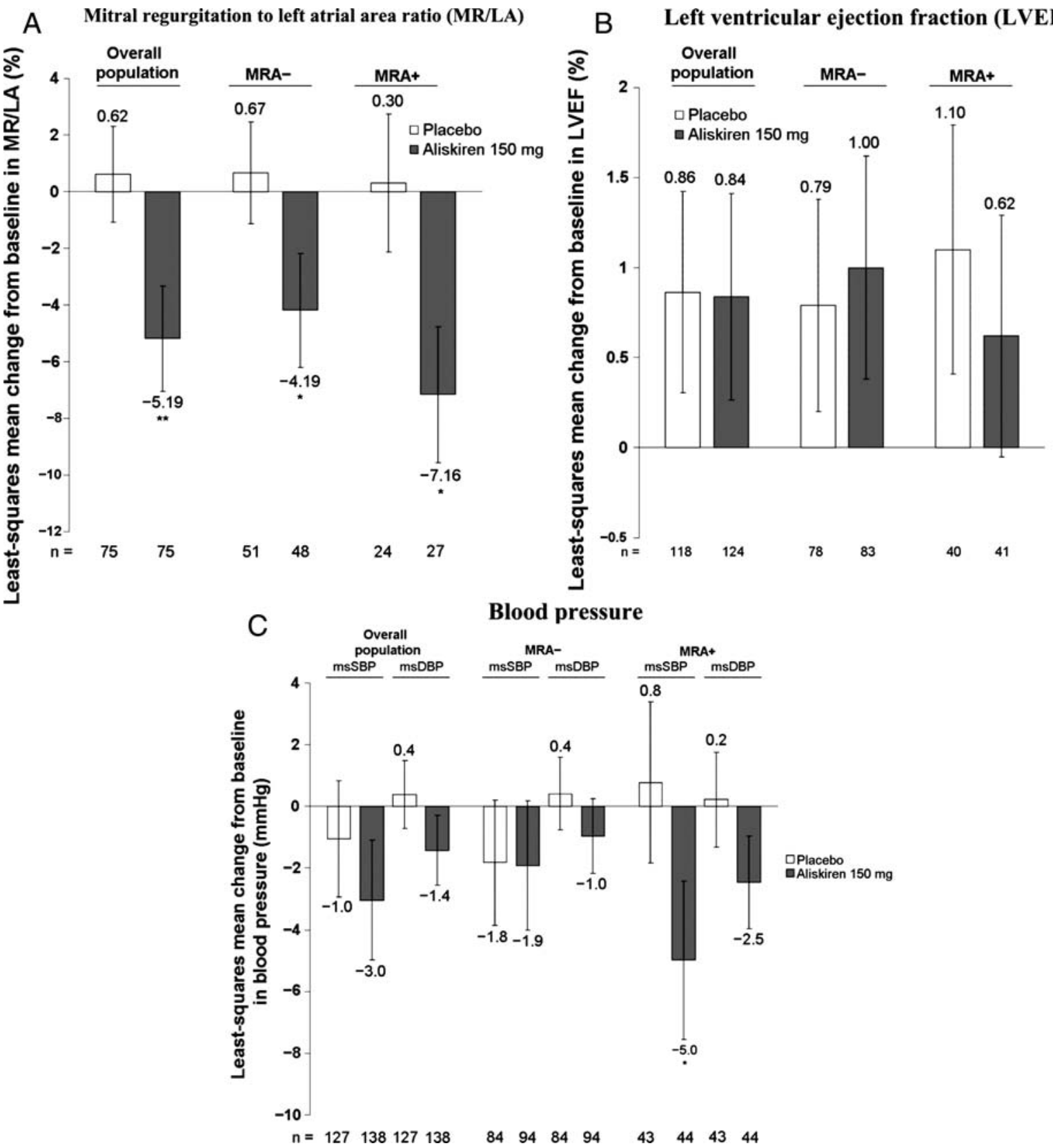

Figure 2 Changes from baseline in echocardiography parameters and blood pressure according to MRA treatment status. (A) Mitral regurgitation to left atrial area ratio, $(B) L V E F$, and $(C)$ blood pressure. Data $(A-C)$ represent least-squares mean $\pm S E M$ in the overall population and the subgroups of patients receiving (MRA+) or not receiving (MRA-) MRA treatment during the study. $P$ values were calculated using a two-way ANCOVA model with treatment, region, LVEF ( $>40$ or $\leq 40 \%)$ and subgroup as factors, baseline as a covariate and treatment by subgroup as an interaction. $* P<0.05$ vs. placebo. ANCOVA, analysis of covariance; Cl, confidence interval; LVEF, left ventricular ejection fraction; MRA, mineralocorticoid receptor antagonist; msDBP, mean sitting diastolic blood pressure; msSBP, mean sitting systolic blood pressure; SEM, standard error of the mean.

\section{Changes in echocardiographic parameters}

In the overall population, aliskiren $150 \mathrm{mg}$ significantly improved the mitral regurgitation to left atrial area ratio (MR/LA) compared with placebo ( -5.2 vs. $+0.6 \%, P=0.0003$; Figure $2 A)$; $M R A$ treatment status did not influence this effect of aliskiren (interaction
$P=0.429 ;$ Figure $3 B$ ). Aliskiren treatment was associated with small numerical increases in LVEF in the overall population and in the MRA - and MRA+ subgroups (Figure 2B), but these changes were not significantly different from those seen with placebo and there was no influence of MRA treatment status (interaction $P=0.409$; Figure $3 B$ ). 


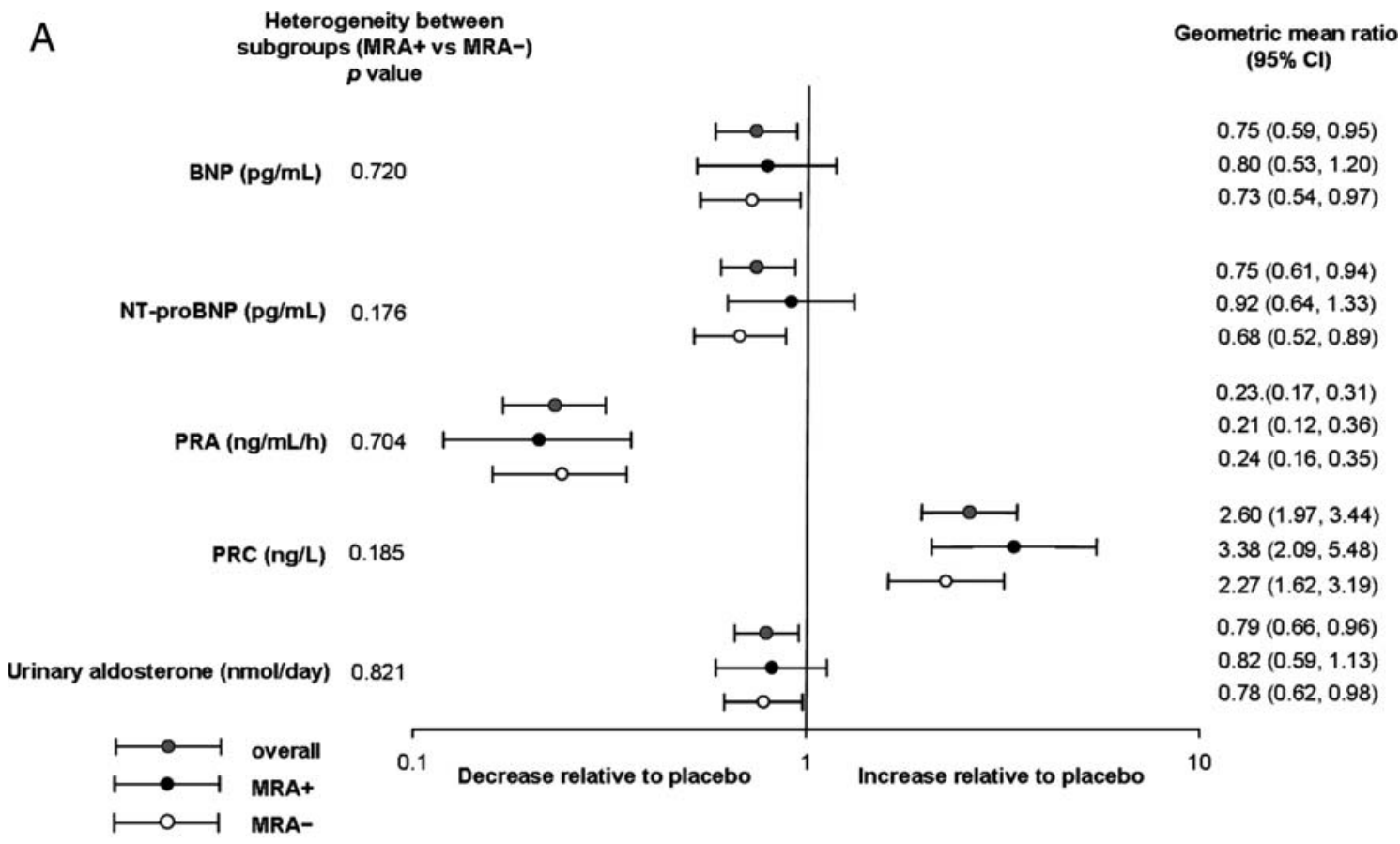

B

Heterogeneity between subgroups (MRA+ vs MRA-)

$p$ value

(MRA+ vs MRA-)

(95\% Cl)

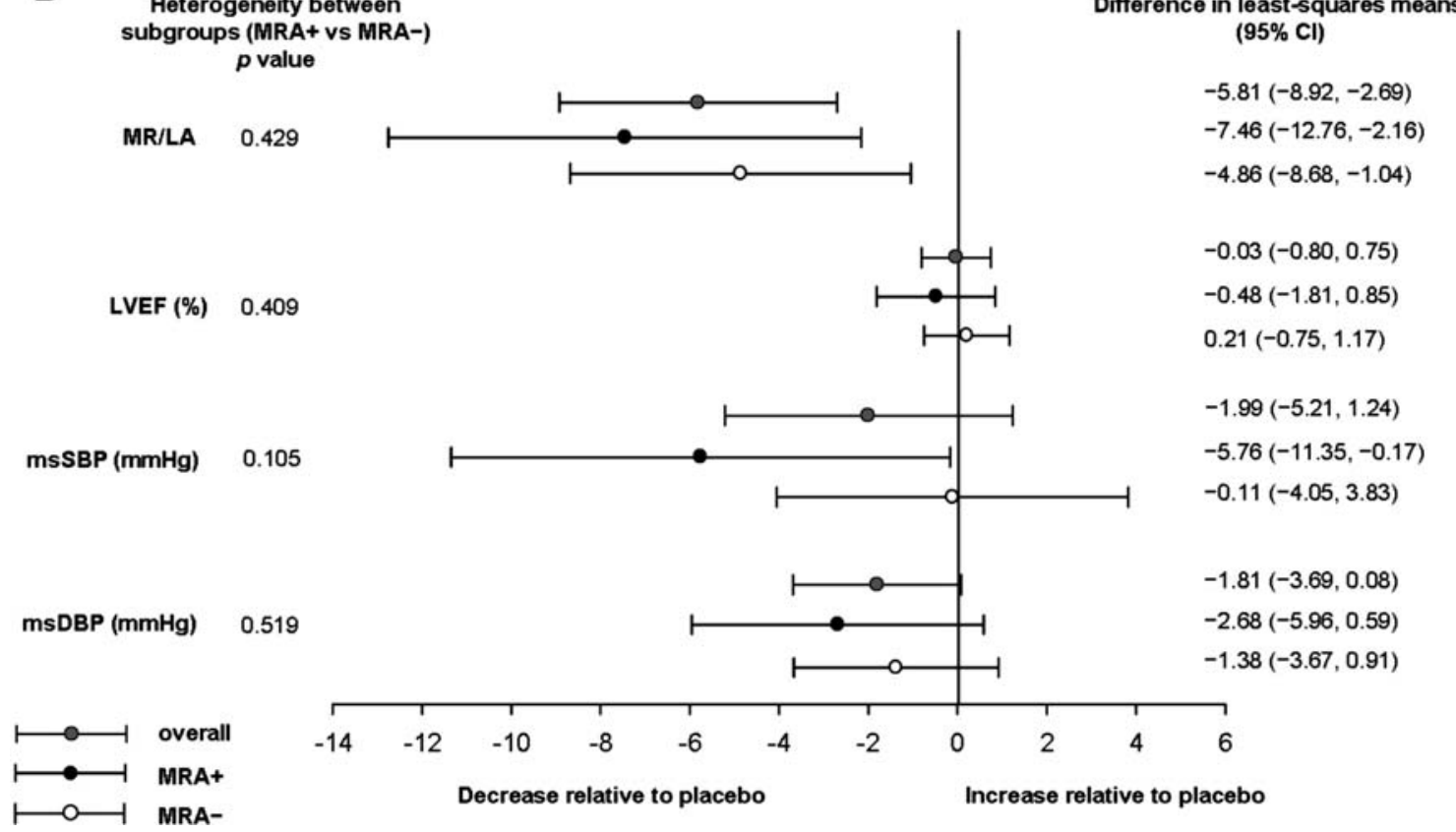

Figure 3 Influence of MRA treatment on between-treatment comparisons (aliskiren vs. placebo) in (A) neurohumoral biomarkers and (B) echocardiography parameters and blood pressure. Data are shown as ratio (aliskiren:placebo) of the geometric mean with associated $95 \%$ confidence intervals (BNP, NT-proBNP, PRA, PRC, urinary aldosterone) or least-squares mean difference (aliskiren - placebo) with associated $95 \%$ confidence intervals (MR/LA, LVEF, blood pressure). $P$ values were calculated using an ANCOVA model with treatment, region, LVEF ( $>40$ or $\leq 40 \%$ ) and subgroup as factors, baseline as a covariate and treatment by subgroup as an interaction. ANCOVA, analysis of covariance; BNP, B-type natriuretic peptide; Cl, confidence interval; LVEF, left ventricular ejection fraction; MRA, mineralocorticoid receptor antagonist; MR/LA, mitral regurgitation to left atrial area ratio; msDBP, mean sitting diastolic blood pressure; msSBP, mean sitting systolic blood pressure; NT-proBNP, N-terminal prohormone B-type natriuretic peptide; PRA, plasma renin activity; PRC, plasma renin concentration. 
Table 3 Safety and tolerability (intent-to-treat population)

\begin{tabular}{|c|c|c|c|c|c|c|c|c|c|}
\hline \multirow{2}{*}{$\begin{array}{l}\text { Pre-specified adverse } \\
\text { event }\end{array}$} & \multicolumn{3}{|c|}{ Overall population } & \multicolumn{3}{|c|}{ Not MRA treated (MRA-) } & \multicolumn{3}{|c|}{ MRA treated (MRA+) } \\
\hline & $\begin{array}{l}\text { Placebo } \\
(N=146)\end{array}$ & $\begin{array}{l}\text { Aliskiren } \\
150 \mathrm{mg} \\
(\mathrm{N}=156)\end{array}$ & $P$-value & $\begin{array}{l}\text { Placebo } \\
(N=97)\end{array}$ & $\begin{array}{l}\text { Aliskiren } \\
150 \mathrm{mg} \\
(n=104)\end{array}$ & $P$-value & $\begin{array}{l}\text { Placebo } \\
(n=49)\end{array}$ & $\begin{array}{l}\text { Aliskiren } \\
150 \mathrm{mg} \\
(n=52)\end{array}$ & $P$-value \\
\hline $\begin{array}{l}\text { Any event of renal } \\
\text { dysfunction, } \\
\text { symptomatic } \\
\text { hypotension, or } \\
\text { hyperkalaemia }\end{array}$ & $11(7.5)$ & $17(10.9)$ & 0.329 & $7(7.2)$ & $8(7.7)$ & 0.898 & $4(8.2)$ & $9(17.3)$ & 0.170 \\
\hline Renal dysfunction & $2(1.4)$ & $3(1.9)$ & 1.000 & $2(2.1)$ & $1(1.0)$ & 0.610 & 0 & $2(3.8)$ & 0.495 \\
\hline $\begin{array}{l}\text { Symptomatic } \\
\text { hypotension }\end{array}$ & $2(1.4)$ & $5(3.2)$ & 0.450 & $1(1.0)$ & $2(1.9)$ & 1.000 & $1(2.0)$ & $3(5.8)$ & 0.618 \\
\hline Hyperkalaemia & $7(4.8)$ & $10(6.4)$ & 0.499 & $4(4.1)$ & $5(4.8)$ & 1.000 & $3(6.1)$ & $5(9.6)$ & 0.716 \\
\hline
\end{tabular}

Table shows the number (\%) of patients experiencing a pre-specified adverse event.

MRA, mineralocorticoid receptor antagonist.

Table 4 Laboratory abnormalities (safety population)

\begin{tabular}{|c|c|c|c|c|c|c|}
\hline \multirow{2}{*}{$\begin{array}{l}\text { Laboratory } \\
\text { abnormalities }\end{array}$} & \multicolumn{2}{|c|}{ Overall population } & \multicolumn{2}{|c|}{ Not MRA treated (MRA-) } & \multicolumn{2}{|c|}{ MRA treated (MRA+) } \\
\hline & $\begin{array}{l}\text { Placebo } \\
(N=146)\end{array}$ & $\begin{array}{l}\text { Aliskiren } 150 \mathrm{mg} \\
(\mathrm{N}=156)\end{array}$ & $\begin{array}{l}\text { Placebo } \\
(N=97)\end{array}$ & $\begin{array}{l}\text { Aliskiren } 150 \mathrm{mg} \\
(\mathrm{N}=104)\end{array}$ & $\begin{array}{l}\text { Placebo } \\
(N=49)\end{array}$ & $\begin{array}{l}\text { Aliskiren } 150 \mathrm{mg} \\
(\mathrm{N}=52)\end{array}$ \\
\hline & {$[n=144]$} & {$[n=156]$} & {$[n=96]$} & {$[n=104]$} & {$[n=48]$} & {$[n=52]$} \\
\hline \multicolumn{7}{|l|}{ Serum potassium } \\
\hline$<3.5 \mathrm{mmol} / \mathrm{L}$ & $7(4.9)$ & $2(1.3)$ & $3(3.1)$ & 0 & $4(8.3)$ & $2(3.8)$ \\
\hline$>5.5 \mathrm{mmol} / \mathrm{L}$ & $12(8.3)$ & $13(8.3)$ & $7(7.3)$ & $6(5.8)$ & $5(10.4)$ & $7(13.5)$ \\
\hline$\geq 6.0 \mathrm{mmol} / \mathrm{L}$ & $6(4.2)$ & $3(1.9)$ & $4(4.2)$ & $2(1.9)$ & $2(4.2)$ & $1(1.9)$ \\
\hline \multicolumn{7}{|l|}{ Serum creatinine } \\
\hline$>177 \mu \mathrm{mol} / \mathrm{L}$ & $8(5.6)$ & $11(7.1)$ & $6(6.3)$ & $8(7.7)$ & $2(4.2)$ & $3(5.8)$ \\
\hline$>265 \mu \mathrm{mol} / \mathrm{L}$ & $3(2.1)$ & 0 & $3(3.1)$ & 0 & 0 & 0 \\
\hline \multicolumn{7}{|l|}{ BUN } \\
\hline$>14.3 \mathrm{mmol} / \mathrm{L}$ & $15(10.4)$ & $13(8.3)$ & $7(7.3)$ & $6(5.8)$ & $8(16.7)$ & $7(13.5)$ \\
\hline
\end{tabular}

Table shows the number (\%) of patients experiencing clinically notable changes in selected laboratory values while receiving treatment.

BUN, blood urea nitrogen; MRA, mineralocorticoid receptor antagonist.

\section{Changes in blood pressure}

In the overall population, there was no significant betweentreatment difference in the change from baseline in msSBP or msDBP (Figure 2C). In the MRA+ subgroup, aliskiren treatment was associated with a significant decrease in msSBP ( -5.0 vs. $+0.8 \mathrm{mmHg}, P<0.05)$ from baseline compared with placebo (Figure 2C). Analysis of covariance revealed no significant interaction between MRA treatment status and the effect of aliskiren on msSBP (interaction $P=0.105$; Figure $3 B$ ) or msDBP (interaction $P=0.519 ;$ Figure $3 B$ ).

\section{Safety and tolerability}

Addition of aliskiren to standard HF treatment was generally well tolerated. There were no significant differences between the aliskiren $150 \mathrm{mg}$ and placebo treatment groups in the proportion of patients who experienced at least one pre-defined adverse event (renal dysfunction, symptomatic hypotension, or hyperkalaemia) in the overall population (Table 3). There were more adverse events with aliskiren compared with placebo in the MRA+ subgroup than in the MRA - one, although the numbers were small and the increase was not statistically significant (Table 3). There were also no notable differences between the aliskiren and placebo groups in the proportion of patients with elevations above pre-defined threshold in blood urea nitrogen, serum creatinine, or serum potassium levels in either the MRA+ or the MRA - subgroups (Table 4).

\section{Discussion}

Recently, the value of adding another blocker of the effects of angiotensin II to an ACE inhibitor in patients with HF has been 
questioned while the indication for using an MRA has broadened to potentially all patients with systolic HF. Specifically, the interpretation of trials adding an ARB to an ACE inhibitor has become controversial. The risk of death from cardiovascular causes was not reduced in each of the two large placebocontrolled trials in HF (although the risk of HF hospitalization was in both). ${ }^{7,8}$ Secondly, the background ACE inhibitor and dose did not match the evidence-based and regulatory standard (i.e. enalapril at an average daily dose of $16.6 \mathrm{mg}$ ). Doubt about the trials in HF has arisen because of the findings of two more recent ARB 'add-on' trials: one in patients with acute myocardial infarction and the other in patients with stable arterial disease. ${ }^{9,10}$ In both, addition of an ARB to a full dose of an evidence-based ACE inhibitor had no clinical benefit. Furthermore, in all of the ARB 'add-on' trials, addition of an ARB to an ACE inhibitor led to increased rates of hypotension, renal dysfunction, and hyperkalaemia. ${ }^{4,5}$

More recently, an unequivocal benefit of an MRA has been shown in patients with systolic HF and mild symptoms, consistent with two other trials with an MRA in systolic HF with severe symptoms and systolic HF after acute myocardial infarction. ${ }^{2,3,11,12}$ MRA were not commonly used at baseline in the aforementioned ARB trials. Consequently, both the efficacy and safety questions about adding a second renin-angiotensin blocker to an ACE inhibitor have changed. In terms of efficacy, it is now important to know whether the second renin-angiotensin blocker provides incremental benefit not only on top of a full dose of an evidence-based ACE inhibitor but also an MRA. This obviously raises a new safety question about adding a second renin-angiotensin blocker not only to an ACE inhibitor but also an MRA (and $\beta$-blocker).

The results of the current post-hoc analysis suggest that the efficacy of aliskiren is maintained in patients receiving an MRA $(M R A+)$ at baseline, as evidenced by a similar reduction in plasma BNP and NT-proBNP to those in the MRA - subgroup. Consistent with the results for the overall population, the present analysis also showed that addition of aliskiren to standard HF therapy led to reductions in urinary aldosterone and PRA levels that were similar in both MRA subgroups. Most importantly, the addition of aliskiren to the MRA + subset was generally well tolerated even though these patients were receiving three RAAS inhibitors (ACE inhibitor or ARB, MRA, and DRI), as well as a $\beta$-blocker in most cases. Specifically, addition of aliskiren to standard HF treatment with or without an MRA was not associated with an increased incidence of pre-specified adverse events including symptomatic hypotension, renal dysfunction, or hyperkalaemia. Although there was a reduction in systolic blood pressure when aliskiren was added to the MRA+ group, this reduction was not significantly different from that observed in the MRA - group. In addition, there were no significant differences between the aliskiren and placebo groups in the proportion of patients with an elevation of blood urea nitrogen, serum creatinine, or serum potassium levels.

Although reductions in the levels of plasma BNP and NT-proBNP have been associated with improved clinical outcomes in HF, these are only surrogate outcomes. ${ }^{13-15}$ Additionally, a post-hoc analysis of one of the ARB 'add-on trials' showed that the benefit of candesartan was consistent irrespective of background use of an MRA. ${ }^{16}$
Both surrogate outcomes and post-hoc analyses can be misleading. ${ }^{17}$ Fortunately, the safety and efficacy of adding aliskiren to conventional therapy in patients with HF is being addressed, appropriately, in an ongoing, large-scale, mortality-morbidity trial (ATMOSPHERE) in which the renin inhibitor is being compared directly with enalapril $10 \mathrm{mg}$ twice daily and also given in addition to enalapril $10 \mathrm{mg}$ twice daily, i.e. a three-arm trial. ${ }^{6}$

In summary, this retrospective analysis of the ALOFT study showed that the natriuretic peptide lowering effect of aliskiren was consistent in patients not treated and treated with an MRA and that there was no excess of renal dysfunction when aliskiren was added to an ACE inhibitor (or ARB) and MRA, as well as a $\beta$-blocker in most cases. However, only the results of a well-powered, prospective, randomized trial will answer the question as to whether the addition of a DRI to an ACE inhibitor (or ARB) and a $\beta$-blocker with or without an MRA will be safe and effective in patients with HF.

\section{Acknowledgements}

All authors participated in the development and writing of the paper, and approved the final manuscript for publication. The authors take full responsibility for the content of the paper and thank Dr Annette Keith for medical writing support, editorial assistance and collation, and incorporation of comments from all authors.

\section{Funding}

This study was funded by Novartis Pharmaceuticals Corporation, East Hanover, NJ, USA.

Conflict of interest: B.A.S., M.W., and M.F.P. are Novartis employees. All other authors or their institutions received funding from Novartis for their participation on the Steering Committee for the ALOFT study and consulting fees, lecture fees, or both from Novartis in relation to aliskiren, other cardiovascular drugs, or both.

\section{References}

1. McMurray JJ, Pitt B, Latini R, Maggioni AP, Solomon SD, Keefe DL, Ford J, Verma A, Lewsey J, Aliskiren Observation of Heart Failure Treatment (ALOFT) Investigators. Effects of the oral direct renin inhibitor aliskiren in patients with symptomatic heart failure. Circ Heart Fail 2008;1:17-24.

2. Zannad F, McMurray J], Drexler H, Krum H, van Veldhuisen DJ, Swedberg K, Shi H, Vincent J, Pitt B. Rationale and design of the Eplerenone in Mild Patients Hospitalization And Survlval Study in Heart Failure (EMPHASIS-HF). Eur J Heart Fail 2010;12:617-622

3. Zannad F, McMurray JJV, Krum H, van Veldhuisen DJ, Swedberg K, Shi H, Vincent J, Drexler H, Pocock S, Pitt B for the the Eplerenone in Mild Patients Hospitalization And Survlval Study in Heart Failure (EMPHASIS-HF) Study. Eplerenone in patients with systolic heart failure and mild symptoms. N Engl J Med 2011;364:11-21

4. Lakhdar R, Al-Mallah MH, Lanfear DE. Safety and tolerability of angiotensinconverting enzyme inhibitor versus the combination of angiotensin-converting enzyme inhibitor and angiotensin receptor blocker in patients with left ventricular dysfunction: a systematic review and meta-analysis of randomized controlled trials. J Card Fail 2008;14:181-188.

5. Desai AS, Swedberg K, McMurray JJ, Granger CB, Yusuf S, Young JB, Dunlap ME, Solomon SD, Hainer JW, Olofsson B, Michelson EL, Pfeffer MA, CHARM Program Investigators. Incidence and predictors of hyperkalemia in patients with heart failure: an analysis of the CHARM Program. J Am Coll Cardiol 2007;50:1959-1966.

6. Krum H, Massie B, Abraham WT, Dickstein K, Kober L, McMurray JJV, Desai A, Gimpelewicz C, Kandra A, Reimun B, Rattunde H, Armbrecht J, on behalf of the ATMOSPHERE Investigators. Direct renin inhibition in addition to or as an alternative to ACE inhibition in patients with systolic chronic heart failure: rationale and design of the Aliskiren Trial to Minimize Outcomes in Patients with Heart failure (ATMOSPHERE) study. Eur J Heart Fail 2011;13:107-114. 
7. Cohn JN, Tognoni G, Valsartan Heart Failure Trial Investigators. A randomized trial of the angiotensin-receptor blocker valsartan in chronic heart failure. $N$ Engl J Med 2001;345:1667-1675.

8. McMurray J], Ostergren J, Swedberg K, Granger CB, Held P, Michelson EL, Olofsson B, Yusuf S, Pfeffer MA, CHARM Investigators and Committees. Effects of candesartan in patients with chronic heart failure and reduced leftventricular systolic function taking angiotensin-converting-enzyme inhibitors: the CHARM-Added trial. Lancet 2003;362:767-771.

9. Pfeffer MA, McMurray JJ, Velazquez EJ, Rouleau JL, Køber L, Maggioni AP, Solomon SD, Swedberg K, Van de Werf F, White H, Leimberger JD, Henis M, Edwards S, Zelenkofske S, Sellers MA, Califf RM, Valsartan in Acute Myocardial Infarction Trial Investigators. Valsartan, captopril, or both in myocardial infarction complicated by heart failure, left ventricular dysfunction, or both. N Engl J Med 2003;349:1893-1906.

10. ONTARGET Investigators, Yusuf S, Teo KK, Pogue J, Dyal L, Copland I, Schumacher H, Dagenais G, Sleight P, Anderson C. Telmisartan, ramipril, or both in patients at high risk for vascular events. N Engl J Med 2008;358: 1547-1559.

11. Pitt B, Zannad F, Remme WJ, Cody R, Castaigne A, Perez A, Palensky J, Wittes J. The effect of spironolactone on morbidity and mortality in patients with severe heart failure. Randomized Aldactone Evaluation Study Investigators. N Engl J Med 1999;341:709-717.

12. Pitt B, Remme W, Zannad F, Neaton J, Martinez F, Roniker B, Bittman R, Hurley S, Kleiman J, Gatlin M, Eplerenone Post-Acute Myocardial Infarction Heart Failure
Efficacy and Survival Study Investigators. Eplerenone, a selective aldosterone blocker, in patients with left ventricular dysfunction after myocardial infarction. N Engl J Med 2003;348:1309-1321.

13. Latini R, Masson S, Anand I, Salio M, Hester A, Judd D, Barlera S, Maggioni AP, Tognoni G, Cohn JN, Val-HeFT Investigators. The comparative prognostic value of plasma neurohormones at baseline in patients with heart failure enrolled in Val-HeFT. Eur Heart J 2004;25:292-299.

14. Latini R, Masson S, Wong M, Barlera S, Carretta E, Staszewsky L, Vago T, Maggioni AP, Anand IS, Tan LB, Tognoni G, Cohn JN, Val-HeFT Investigators. Incremental prognostic value of changes in B-type natriuretic peptide in heart failure. Am J Med 2006;119:70.e23-30.

15. Masson S, Latini R, Anand IS, Barlera S, Angelici L, Vago T, Tognoni G, Cohn JN, $\mathrm{Val}-\mathrm{HeFT}$ Investigators. Prognostic value of changes in $\mathrm{N}$-terminal pro-brain natriuretic peptide in Val-HeFT (Valsartan Heart Failure Trial). J Am Coll Cardiol 2008;52:997-1003.

16. Weir RA, McMurray JJ, Puu M, Solomon SD, Olofsson B, Granger CB, Yusuf S, Michelson EL, Swedberg K, Pfeffer MA, CHARM Investigators. Efficacy and tolerability of adding an angiotensin receptor blocker in patients with heart failure already receiving an angiotensin-converting inhibitor plus aldosterone antagonist, with or without a beta blocker. Findings from the Candesartan in Heart failure: Assessment of Reduction in Mortality and morbidity (CHARM)-Added trial. Eur J Heart Fail 2008;10:157-163.

17. Granger CB, McMurray JJ. Using measures of disease progression to determine therapeutic effect: a sirens' song. J Am Coll Cardiol 2006;48:434-437. 